\title{
Embedding theorems for anisotropic Lipschitz spaces
}

\author{
by \\ F. J. PÉREZ (Logroño)
}

\begin{abstract}
Anisotropic Lipschitz spaces are considered. For these spaces we obtain sharp embeddings in Besov and Lorentz spaces. The methods used are based on estimates of iterative rearrangements. We find a unified approach that arises from the estimation of functions defined as minimum of a given system of functions. The case of $L^{1}$-norm is also covered.
\end{abstract}

1. Introduction. In this paper we prove embedding theorems for anisotropic Lipschitz spaces. More precisely, we study integrability and smoothness properties of functions under certain conditions on their moduli of continuity.

In the study of anisotropic spaces, we have different estimates with respect to different variables. The final result will be sharp if we find a balance between these estimates, that is, an optimal average estimate. Therefore it is an important problem to determine a right contribution of each variable to this average. To discuss this problem we first recall some basic definitions.

Denote by $W_{p ; j}^{r}\left(\mathbb{R}^{n}\right)(r \in \mathbb{N}, 1 \leq p<\infty, 1 \leq j \leq n)$ the Sobolev space with respect to the $j$ th variable, i.e. the class of functions $f$ in $L^{p}\left(\mathbb{R}^{n}\right)$ with generalized partial derivative $D_{j}^{r} f \in L^{p}\left(\mathbb{R}^{n}\right)$. Now, if $r_{1}, \ldots, r_{n} \in \mathbb{N}$, then we set

$$
W_{p}^{r_{1}, \ldots, r_{n}}\left(\mathbb{R}^{n}\right) \equiv \bigcap_{j=1}^{n} W_{p ; j}^{r_{j}}\left(\mathbb{R}^{n}\right)
$$

and

$$
\|f\|_{W_{p}^{r_{1}, \ldots, r_{n}}}=\|f\|_{p}+\sum_{j=1}^{n}\left\|D_{j}^{r_{j}} f\right\|_{p} .
$$

2000 Mathematics Subject Classification: Primary 46E35, 46E30.

Thanks to V. I. Kolyada for his useful ideas and suggestions, work and time dedicated. Research supported in part by grant BMF2003-06335-C03-03 of the DGI, Spain.

This paper was completed when the author worked at the Institute of Mathematics of the Polish Academy of Sciences under a "Marie Curie Training Site" fellowship. 
If $f$ is a function on $\mathbb{R}^{n}, 1 \leq j \leq n$, and $k \in \mathbb{N}$, then we define

$$
\Delta_{j}^{k}(h) f(x)=\sum_{i=0}^{k}(-1)^{k-i}\left(\begin{array}{l}
k \\
i
\end{array}\right) f\left(x+i h e_{j}\right)
$$

(where $x \in \mathbb{R}^{n}, h \in \mathbb{R}$, and $e_{j}$ is a basis vector). Let $f \in L^{p}\left(\mathbb{R}^{n}\right)(1 \leq p<\infty)$. Then the function

$$
\omega_{j}^{k}(f ; \delta)_{p}=\sup _{0 \leq h \leq \delta}\left\|\Delta_{j}^{k}(h) f\right\|_{p} \quad(\delta \geq 0)
$$

is called the partial modulus of continuity of $f$ of order $k$ in $L^{p}$ with respect to $x_{j}$.

Let $r>0$, and let $k$ be the least integer such that $k>r$. We denote by $H_{p ; j}^{r}\left(\mathbb{R}^{n}\right)$ the Nikol'skiu space of functions $f$ in $L^{p}\left(\mathbb{R}^{n}\right)$ for which

$$
\omega_{j}^{k}(f ; \delta)_{p}=O\left(\delta^{r}\right) .
$$

Assume that $r_{j}>0(j=1, \ldots, n)$ and that $k_{j}$ is the least integer such that $k_{j}>r_{j}$. Then $H_{p}^{r_{1}, \ldots, r_{n}}\left(\mathbb{R}^{n}\right)$ is defined as $\bigcap_{j=1}^{n} H_{p ; j}^{r_{j}}\left(\mathbb{R}^{n}\right)$, with the norm

$$
\|f\|_{H_{p}^{r_{1}, \ldots, r_{n}}}=\|f\|_{p}+\sum_{j=1}^{n} \sup _{u>0} u^{-r_{j}} \omega_{j}^{k_{j}}(f ; u)_{p} .
$$

It is well known that an important characteristic of the $W$ spaces is the harmonic mean

$$
r=n\left(\sum_{j=1}^{n} \frac{1}{r_{j}}\right)^{-1}
$$

(see $[9,10])$. In particular, if $1 \leq p<n / r$ and $q^{*}=n p /(n-r p)$, then

$$
W_{p}^{r_{1}, \ldots, r_{n}} \hookrightarrow L^{q}
$$

if and only if $p \leq q \leq q^{*}$. That is, the integrability properties of functions in $W_{p}^{r_{1}, \ldots, r_{n}}$ are completely determined by $r$, and the contribution of the variable $x_{k}$ is proportional to $1 / r_{k}$ in a sense. A similar situation holds for Nikol'skil spaces, although in this case the embedding with the limit exponent fails (see $[15,2]$ ).

However, the behaviour of anisotropic Lipschitz spaces is completely different.

Let $1 \leq p<\infty, r>0$ and let $\bar{r}$ be the least integer such that $\bar{r} \geq r$. We say that $f \in L^{p}\left(\mathbb{R}^{n}\right)$ belongs to the Lipschitz space $\Lambda_{p ; j}^{r}\left(\mathbb{R}^{n}\right)$ with respect to the $j$ th variable if

$$
\omega_{j}^{\bar{r}}(f ; \delta)_{p}=O\left(\delta^{r}\right)
$$

Let $r_{j}>0(j=1, \ldots, n)$ and denote by $\bar{r}_{j}$ the least integer with $r_{j} \leq \bar{r}_{j}$. The anisotropic Lipschitz space $\Lambda_{p}^{r_{1}, \ldots, r_{n}}\left(\mathbb{R}^{n}\right)$ is defined as $\bigcap_{j=1}^{n} \Lambda_{p ; j}^{r_{j}}\left(\mathbb{R}^{n}\right)$. So,

$$
\|f\|_{\Lambda_{p}^{r_{1}, \ldots, r_{n}}} \equiv\|f\|_{p}+\|f\|_{\lambda_{p}^{r_{1}, \ldots, r_{n}}}
$$


where the seminorm is

$$
\|f\|_{\lambda_{p}^{r_{1}, \ldots, r_{n}}}=\sum_{j=1}^{n} \sup _{\delta>0} \delta^{-r_{j}} \omega_{j}^{\bar{r}_{j}}(f ; \delta)_{p} .
$$

It is clear that

$$
\Lambda_{p ; j}^{r_{j}}=H_{p ; j}^{r_{j}} \quad \text { if } r_{j} \notin \mathbb{N} .
$$

Also, by the Hardy-Littlewood theorem [15], if $r_{j} \in \mathbb{N}$, then

$$
\Lambda_{p ; j}^{r_{j}}=W_{p ; j}^{r_{j}} \quad(p>1) .
$$

For $r_{j} \in \mathbb{N}$ we have the strict embedding $\Lambda_{p ; j}^{r_{j}} \subset H_{p ; j}^{r_{j}}$.

Thus, Lipschitz spaces have partly the character of Sobolev spaces, and partly of Nikol'skiı spaces. This mixed behaviour creates the main difficulty in their study.

Integrability properties of functions in a Lipschitz space and Nikol'skiu space with the same indices can be completely different. It was proved in [5] (for $r_{k} \leq 1$ ) that, in contrast to $W$ and $H$ spaces, the embedding $\Lambda_{p}^{r_{1}, \ldots, r_{n}} \hookrightarrow L^{q}$ is not uniquely determined by the value of the harmonic mean $r$ (see (1.1)). Roughly speaking, this means that the contribution of the variable $x_{k}$ is not proportional to $1 / r_{k}$.

The proof in [5] (as well as alternative proofs given in [7, 8]) was based on estimates of rearrangements and special reasonings that led to a kind of balance between these estimates.

One of the main objectives of this paper is to give a quantitative sharp expression for this type of balance. We obtain the following results. First, basing on known estimates of rearrangements, we modify them to special ones involving functions from the spaces $L^{\theta}\left(\mathbb{R}_{+}, d x / x\right), \mathbb{R}_{+} \equiv(0, \infty)$. The invariance of these spaces under changes of variables of power type plays an important role. Then, using the modified estimates, we consider the "minimum-function"

$$
\begin{aligned}
\varrho(t)=\min _{1 \leq i \leq n}\left\{t_{i}^{r_{i}} \phi_{i}\left(t_{l_{i}}\right)\right\} & \\
\qquad & \quad \in \mathbb{R}_{+}^{n}, \phi_{i} \in L^{\theta_{i}}\left(\mathbb{R}_{+}, d x / x\right), l_{i} \in\{1, \ldots, n\} .
\end{aligned}
$$

We prove a special weight estimate for this function. This provides a unified approach to estimations of various norms. Using this approach, we prove sharp estimates of Lorentz norms as well as Besov norms for functions in Lipschitz spaces.

Let us give a more detailed description of the latter results.

As mentioned above, the first sharp results on embedding of Lipschitz spaces into $L^{q}$ were obtained in [5] (for $r_{k} \leq 1$ ) with the use of nonincreasing rearrangements. Afterwards, Netrusov [13, 14] studied embeddings of $\Lambda_{p}^{r_{1}, \ldots, r_{n}}$ for $p>1$ and arbitrary $r_{j}>0$. His approach was based on 
special integral representations. First, he proved sharp results on embedding into Lorentz spaces (an alternative proof of those results including the case $p=1$ was given in [10] and was based on non-increasing rearrangements). Then he considered embedding into Besov spaces.

Assume that $1 \leq p, \theta_{j}<\infty$ and $0<r_{j}<\infty(j=1, \ldots, n)$. The anisotropic Besov space $B_{p, \theta_{1}, \ldots, \theta_{n}}^{r_{1}, \ldots, r_{n}}\left(\mathbb{R}^{n}\right)$ is the class of functions $f \in L^{p}\left(\mathbb{R}^{n}\right)$ such that

$$
\|f\|_{B_{p, \theta_{1}, \ldots, \theta_{n}}^{r_{1}, \ldots, r_{n}}} \equiv\|f\|_{p}+\sum_{j=1}^{n}\left(\int_{0}^{\infty}\left(t^{-r_{j}}\left\|\Delta_{j}^{k_{j}}(t) f\right\|_{p}\right)^{\theta_{j}} \frac{d t}{t}\right)^{1 / \theta_{j}}<\infty,
$$

where $k_{j} \in \mathbb{N}$ and $k_{j}>r_{j}$. For each choice of the integers $k_{j}$ one obtains equivalent norms; in addition, one can replace the norm of finite differences by the corresponding moduli of continuity ([15, Chapter 4] and [2, Chapter 4]). For simplicity we write $B_{p, \theta}^{r_{1}, \ldots, r_{n}} \equiv B_{p, \theta, \ldots, \theta}^{r_{1}, \ldots, r_{n}}$.

Il'in $[2, \S 18.12]$ obtained the following refinement of the classical Sobolev inequality: if $1<p<q<\infty, r_{j} \in \mathbb{N}$, and $\varkappa \equiv 1-(n / r)(1 / p-1 / q)>0$, then

$$
W_{p}^{r_{1}, \ldots, r_{n}}\left(\mathbb{R}^{n}\right) \hookrightarrow B_{q, p}^{\varkappa r_{1}, \ldots, \varkappa r_{n}}\left(\mathbb{R}^{n}\right) .
$$

In the case $p=n=1$ this embedding fails. It was proved by $\operatorname{Kolyada}[6,9]$ that the embedding (1.3) is also true in the case $p=1, n \geq 2$.

For Lipschitz spaces, the following result was obtained by Netrusov [14]: if $1<p<q<\infty, r_{j}>0(j=1, \ldots, n)$, and $\varkappa \equiv 1-(n / r)(1 / p-1 / q)>0$, then

$$
\Lambda_{p}^{r_{1}, \ldots, r_{n}}\left(\mathbb{R}^{n}\right) \hookrightarrow B_{q, \gamma_{1}, \ldots, \gamma_{n}}^{\alpha_{1}, \ldots, \alpha_{n}}\left(\mathbb{R}^{n}\right) .
$$

Although we do not specify here the values of the parameters, it is important to point out that here $\gamma_{j}$ takes two values: one for all $j$ such that $r_{j} \notin \mathbb{N}$ and another one in the case $r_{j} \in \mathbb{N}$.

Let us emphasize that the methods of integral representations used in [14] fail in the case $p=1$. In particular, the question on validity of the embedding for $p=1$ has remained open.

In this paper ( $(5)$ we prove the embedding (1.4) for $p \geq 1$. It is the most important application of our main estimates concerning integrability of functions of type (1.2). Moreover, we prove estimates for stronger norms defined in terms of iterative rearrangements.

For a given function on $\mathbb{R}^{n}$, we obtain its iterative rearrangement by rearranging the function first with respect to one variable, then another, and so on. It turns out that the iterative rearrangement is defined on $\mathbb{R}_{+}^{n} \equiv(0, \infty)^{n}$, it is non-increasing in each variable and equimeasurable with $|f|$. A Lorentz kind norm $\|\cdot\|_{q, p ; \mathcal{R}}$ can be defined in terms of iterative rearrangements (see $\S 2)$. It is important to stress that in the case $q>p$ this norm is stronger than the usual Lorentz norm $\|\cdot\|_{q, p}$. Observe also that iterative rearrange- 
ments were used in embedding theorems in $[5,6,8,11,16]$. In particular, it was proved in [11] that for anisotropic Sobolev spaces a stronger version of Sobolev type inequality with the generalized Lorentz norm $\|\cdot\|_{q, p ; \mathcal{R}}$ is true.

Applying estimates of functions (1.2) we immediately obtain a similar result for Lipschitz spaces. That is, in Section 5 we prove a Sobolev type inequality

$$
\|f\|_{q^{*}, s ; \mathcal{R}} \leq c\|f\|_{\lambda_{p}^{r_{1}, \ldots, r_{n}}}, \quad 1 \leq p<n / r
$$

which gives an extension of the results of Kolyada and Netrusov mentioned above.

Further, in Section 5 we prove one of our main results, Il'in's type inequality

$$
\sum_{i=1}^{n}\left(\int_{0}^{\infty}\left[h^{-\alpha_{i}}\left\|\Delta_{i}^{\bar{r}_{i}}(h) f\right\|_{q, 1 ; \mathcal{R}}\right]^{\gamma_{i}} \frac{d h}{h}\right)^{1 / \gamma_{i}} \leq c\|f\|_{\lambda_{p}^{r_{1}, \ldots, r_{n}}} .
$$

This immediately implies the embedding (1.4) for all $p \geq 1$. Let us emphasize that $p=1$ is included. Moreover, comparing with (1.4), the left hand side of (1.5) contains the stronger Lorentz norm $\|\cdot\|_{q, 1 ; \mathcal{R}}$ instead of $\|\cdot\|_{q}$. Note also that it is even possible to replace $\|\cdot\|_{q, 1 ; \mathcal{R}}$ by a stronger norm $\|\cdot\|_{q, \xi ; \mathcal{R}}$ for any $\xi>0$.

As mentioned above, our approach is based on two tools. First, we use some modifications of estimates of rearrangements obtained in $[11,10]$. Second, we apply estimates of functions of type (1.2).

The paper is organized as follows. In Section 2 we consider the definition and basic properties of iterative rearrangements. Section 3 is devoted to modifying known estimates of rearrangements into a special type. Next, in Section 4 we get the main lemmas that give us special weight estimates for functions of type (1.2). Finally, sharp embeddings for anisotropic Lipschitz spaces are proved in Section 5.

2. Non-increasing rearrangements. This section contains basic facts concerning rearrangements. We refer to $[11, \S 2]$ for details.

Let $S_{0}\left(\mathbb{R}^{n}\right)$ be the class of measurable and almost everywhere finite functions $f$ on $\mathbb{R}^{n}$ such that for each $y>0$,

$$
\lambda_{f}(y) \equiv\left|\left\{x \in \mathbb{R}^{n}:|f(x)|>y\right\}\right|<\infty .
$$

A non-increasing rearrangement of a function $f \in S_{0}\left(\mathbb{R}^{n}\right)$ is a non-increasing function $f^{*}$ on $\mathbb{R}_{+} \equiv(0, \infty)$ that is equimeasurable with $|f|$. The rearrangement $f^{*}$ can be defined by the equality

$$
f^{*}(t)=\sup _{|E|=t} \inf _{x \in E}|f(x)|, \quad 0<t<\infty .
$$

Next, we consider the so-called iterative rearrangements. 
Let $x=\left(x_{1}, \ldots, x_{n}\right) \in \mathbb{R}^{n}$. Removing the variable $x_{k}$ from the $n$-tuple $x$ we obtain an $(n-1)$-dimensional vector denoted by $\widehat{x}_{k}$.

We denote by $\left(\tau, \widehat{x}_{k}\right)(\tau \in \mathbb{R})$ the vector in $\mathbb{R}^{n}$ with first component $\tau$ and the remaining components equal to the $(n-1)$-dimensional vector $\widehat{x}_{k}$.

Let $k \in\{1, \ldots, n\}$ and $f \in S_{0}\left(\mathbb{R}^{n}\right)$. We obtain $\mathcal{R}_{k} f\left(t_{1}, \widehat{x}_{k}\right)$ a.e. on $\mathbb{R}_{+} \times$ $\mathbb{R}^{n-1}$ by fixing $\widehat{x}_{k}$ and "rearranging" $f$ in non-increasing order as a function of the variable $x_{k}$ only.

Let $\mathcal{P}_{n}$ be the collection of all permutations $\sigma=\left\{k_{1}, \ldots, k_{n}\right\}$ of the set $\{1, \ldots, n\}$. For each $\sigma \in \mathcal{P}_{n}$ we set $\mathcal{R}_{\sigma} f \equiv \mathcal{R}_{k_{n}} \cdots \mathcal{R}_{k_{1}} f$. It is easy to see that $\mathcal{R}_{\sigma} f$ decreases with respect to each variable and is equimeasurable with $|f|$ (for more details, see $[11, \S 2]$ ).

It is easy to verify that

$$
\begin{gathered}
\mathcal{R}_{\sigma} f(t) \leq f^{*}\left(t_{1} \cdots t_{n}\right), \\
\mathcal{R}_{\sigma}(f+g)(t+s) \leq \mathcal{R}_{\sigma} f(t)+\mathcal{R}_{\sigma} g(s) \quad\left(t, s \in \mathbb{R}_{+}^{n}\right) .
\end{gathered}
$$

Let $k \in\{1, \ldots, n\}, t_{1} \in \mathbb{R}_{+}$, and $\widehat{x}_{k} \in \mathbb{R}^{n-1}$. We consider the following averages:

$$
\mathcal{R}_{k}^{*} f\left(t_{1}, \widehat{x}_{k}\right) \equiv \frac{1}{t_{1}} \int_{0}^{t_{1}} \mathcal{R}_{k} f\left(u, \widehat{x}_{k}\right) d u, \quad \overline{\mathcal{R}}_{k} f\left(t_{1}, \widehat{x}_{k}\right) \equiv \frac{1}{t_{1}} \int_{t_{1}}^{\infty} \mathcal{R}_{k} f\left(u, \widehat{x}_{k}\right) d u .
$$

Now, for each $\sigma \in \mathcal{P}_{n}$ we set

Then (see $[11, \S 2])$

$$
\mathcal{R}_{\sigma}^{*} f(t)=\mathcal{R}_{k_{n}}^{*} \cdots \mathcal{R}_{k_{1}}^{*} f(t), \quad t \in \mathbb{R}_{+}^{n} .
$$

$$
\left\|\mathcal{R}_{\sigma}^{*} f\right\|_{p} \leq c_{p}\|f\|_{p}, \quad 1<p<\infty
$$

We also define

$$
\overline{\mathcal{R}}_{\sigma} f(t)=\overline{\mathcal{R}}_{k_{n}} \cdots \overline{\mathcal{R}}_{k_{1}} f(t), \quad t \in \mathbb{R}_{+}^{n},
$$

and for each $1<\nu<\infty$ we set

$$
\overline{\mathcal{R}}_{\sigma}^{(\nu)} f(t) \equiv\left(\overline{\mathcal{R}}_{\sigma} f^{\nu}(t)\right)^{1 / \nu} .
$$

This operator was defined in [11] and used to prove embedding theorems. It has the important property that

$$
\left\|\overline{\mathcal{R}}_{\sigma}^{(\nu)} f\right\|_{1} \leq c\|f\|_{1} .
$$

Assume that $0<p, q<\infty$. A function $f \in S_{0}\left(\mathbb{R}^{n}\right)$ belongs to the Lorentz space $L^{q, p}\left(\mathbb{R}^{n}\right)$ if

$$
\|f\|_{q, p} \equiv\left(\int_{0}^{\infty}\left(t^{1 / q} f^{*}(t)\right)^{p} \frac{d t}{t}\right)^{1 / p}<\infty .
$$

We have the inequality (see $[1$, p. 217])

$$
\|f\|_{q, s} \leq c\|f\|_{q, p} \quad(0<p \leq s<\infty),
$$


so that $L^{q, p} \subset L^{q, s}$ for $p<s$. In particular, for $0<p \leq q$,

$$
L^{q, p} \subset L^{q, q} \equiv L^{q} .
$$

In what follows we set

$$
\pi(t)=\prod_{k=1}^{n} t_{k}, \quad t \in \mathbb{R}_{+}^{n} .
$$

Assume that $0<q, p<\infty$ and let $\sigma \in \mathcal{P}_{n}(n \geq 2)$. We denote by $L_{\mathcal{R}_{\sigma}}^{q, p}\left(\mathbb{R}^{n}\right)$ the class of functions $f \in S_{0}\left(\mathbb{R}^{n}\right)$ such that

$$
\|f\|_{q, p ; \mathcal{R}_{\sigma}} \equiv\left(\int_{\mathbb{R}_{+}^{n}}\left[\pi(t)^{1 / q_{\mathcal{R}_{\sigma}}} f(t)\right]^{p} \frac{d t}{\pi(t)}\right)^{1 / p}<\infty
$$

(see [3]). We also set

$$
L_{\mathcal{R}}^{q, p}\left(\mathbb{R}^{n}\right)=\bigcap_{\sigma \in \mathcal{P}_{n}} L_{\mathcal{R}_{\sigma}}^{q, p}\left(\mathbb{R}^{n}\right), \quad\|f\|_{q, p ; \mathcal{R}}=\sum_{\sigma \in \mathcal{P}_{n}}\|f\|_{q, p ; \mathcal{R}_{\sigma}} .
$$

It is easy to see that

$$
\|f\|_{q, s ; \mathcal{R}} \leq c\|f\|_{q, p ; \mathcal{R}} \quad(0<p \leq s<\infty) .
$$

If $q>p$, then for each $\sigma \in \mathcal{P}_{n}$ and each $f \in S_{0}\left(\mathbb{R}^{n}\right)$,

$$
\|f\|_{q, p} \leq c\|f\|_{q, p ; \mathcal{R}_{\sigma}}
$$

(see [17]). Thus,

$$
L_{\mathcal{R}_{\sigma}}^{q, p} \subset L^{q, p} \quad(q>p) .
$$

Moreover, this is a proper embedding [17].

3. Estimates. From now on $n \in \mathbb{N}$. Let $0<r_{j}<\infty(j=1, \ldots, n)$. We denote by $\bar{r}_{j}$ the least integer such that $r_{j} \leq \bar{r}_{j}$.

Let $f \in L^{p}\left(\mathbb{R}^{n}\right)(1 \leq p<\infty)$. For each $j=1, \ldots, n$ set

$$
f_{j, h}(x) \equiv \Delta_{j}^{\bar{r}_{j}}(h) f(x) .
$$

In this section we consider some modifications of the estimates of the iterative rearrangements $\mathcal{R}_{\sigma} f$ and $\mathcal{R}_{\sigma} f_{j, h}$ obtained in [11] and [10].

For $1 \leq p<\infty$ we write $\mathcal{L}^{p} \equiv L^{p}\left(\mathbb{R}_{+}, d u / u\right)$; set also $\mathcal{L}^{\infty} \equiv L^{\infty}\left(\mathbb{R}_{+}\right)$ (see [4]).

Lemma 1. Let $n \in \mathbb{N}$ and $1 \leq p<\infty$. Assume that $F \in L^{p}\left(\mathbb{R}_{+}^{n}\right)$ is a non-negative function, non-increasing in each of its variables. Then for any $\delta>0$ and any $j \in\{1, \ldots, n\}$ there exists a non-negative function $\phi \equiv \phi_{\delta, j}$ on $\mathbb{R}_{+}$such that

(i) $F(t) \leq \pi(t)^{-1 / p} \phi\left(t_{j}\right)$,

(ii) $\|\phi\|_{\mathcal{L}^{p}} \leq c(\delta)\|F\|_{L^{p}\left(\mathbb{R}_{+}^{n}\right)}$,

(iii) $\phi(u) u^{\delta} \uparrow$ and $\phi(u) u^{-\delta} \downarrow$. 
Proof. As $F$ is non-increasing in each variable, we use a weak type inequality

$$
F(t) \leq \pi\left(\widehat{t}_{j}\right)^{-1 / p}\left(\int_{\mathbb{R}_{+}^{n-1}} F(t)^{p} d \widehat{t}_{j}\right)^{1 / p} \equiv \pi\left(\widehat{t}_{j}\right)^{-1 / p} g\left(t_{j}\right) .
$$

Then $g$ is non-negative and non-increasing in $\mathbb{R}_{+}$and

$$
\|g\|_{L^{p}\left(\mathbb{R}_{+}\right)}=\|F\|_{L^{p}\left(\mathbb{R}_{+}^{n}\right)} .
$$

Applying Lemma 2.1 of [12] we obtain a function $\bar{g}$ on $\mathbb{R}_{+}$such that

$$
\text { (3.3) } g \leq \bar{g}, \quad\|\bar{g}\|_{p} \leq c(\delta)\|g\|_{p}, \quad \bar{g}(u) u^{1 / p-\delta} \downarrow, \bar{g}(u) u^{1 / p+\delta} \uparrow, \quad u>0 .
$$

Setting $\phi(u) \equiv \bar{g}(u) u^{1 / p}$, by (3.1) and (3.3) we get (i). Next, (ii) follows from (3.2) and (3.3), and (iii) follows from (3.3).

Lemma 2. Let $n \geq 2, j \in\{1, \ldots, n\}, r_{j} \in \mathbb{N}$ and $1 \leq p<\infty$. Let $f \in W_{p ; j}^{r_{j}}\left(\mathbb{R}^{n}\right)$. Choose $\sigma \in \mathcal{P}_{n}, 1 \leq l \leq n\left(l \neq \sigma^{-1}(j)\right)$, and $0<\delta<1$. Then there exists a non-negative function $\phi \equiv \phi_{j, l, \sigma, \delta}$ on $\mathbb{R}_{+}$such that:

$$
\begin{gathered}
\|\phi\|_{\mathcal{L}^{p}} \leq c\left\|D_{j}^{r_{j}} f\right\|_{p} ; \\
\phi(u) u^{\delta \uparrow} \text { and } \phi(u) u^{-\delta} \downarrow, \quad u>0 ;
\end{gathered}
$$

for any $K>1$,

$$
\begin{aligned}
& \mathcal{R}_{\sigma} f(t) \leq 2^{r_{j}} \mathcal{R}_{\sigma_{j}^{\prime}} f\left(K t_{m_{j}}, \widehat{t}_{m_{j}} / 2\right)+c(K) \pi(t)^{-1 / p} t_{m_{j}}^{r_{j}} \phi\left(t_{l}\right) ; \\
& \mathcal{R}_{\sigma} f_{j, h}(t) \leq c \pi(t)^{-1 / p} h^{r_{j}-\delta} t_{m_{j}}^{\delta} \phi\left(t_{l}\right) \quad \text { for all } 0<h<t_{m_{j}},
\end{aligned}
$$

where $\sigma_{j}^{\prime}$ is obtained from $\sigma$ by moving the $j$ th index to the first position, $m_{j}=\sigma^{-1}(j)$, and $c, c(K)$ do not depend on $f$.

Proof. CaSE 1: $p>1$. Set $g_{j} \equiv D_{j}^{r_{j}} f$. From [11, (3.3) and (3.7)] we get

$$
\begin{aligned}
\mathcal{R}_{\sigma} f(t) \leq & 2^{r_{j}} \mathcal{R}_{\sigma_{j}^{\prime}} f\left(K t_{m_{j}}, \widehat{t}_{m_{j}} / 2\right) \\
& +c(K) t_{m_{j}}^{r_{j}} \mathcal{R}_{\sigma}^{*} g_{j}(t / 2) \quad \text { for all } K>1,
\end{aligned}
$$

where $\sigma_{j}^{\prime}$ is obtained from $\sigma$ by moving the $j$ th index to the first position.

Moreover, by [11, (4.5)],

$$
\mathcal{R}_{\sigma} f_{j, h}(t) \leq c h^{r_{j}} \mathcal{R}_{\sigma}^{*} g_{j}(t) .
$$

Now (see $(2.2))$ note that $\mathcal{R}_{\sigma}^{*} g_{j}(t)$ satisfies the conditions of Lemma 1. So, for $\delta$ and $l$ we obtain a non-negative function $\phi$ such that (3.5) holds,

$$
\mathcal{R}_{\sigma}^{*} g_{j}(t) \leq \pi(t)^{-1 / p} \phi\left(t_{l}\right)
$$

and

$$
\|\phi\|_{\mathcal{L}^{p}} \leq c\left\|\mathcal{R}_{\sigma}^{*} g_{j}\right\|_{p}
$$

Then (3.4) follows from the last estimate and (2.2). Inequalities (3.6) and (3.7) are immediate consequences of (3.10), (3.8) and (3.9). 
CASE $2: p=1$. Set $\nu=1 /(1-\delta)$. We have (see [11, (3.3) and (3.10)])

$$
\mathcal{R}_{\sigma} f(t) \leq 2^{r_{j}} \mathcal{R}_{\sigma_{j}^{\prime}} f\left(K t_{m_{j}}, \widehat{t}_{m_{j}} / 2\right)+c(K) t_{m_{j}}^{r_{j}-1} F_{j}\left(\widehat{t}_{m_{j}} / 2\right),
$$

where

$$
F_{j}\left(\widehat{t}_{m_{j}}\right)=\overline{\mathcal{R}}_{\widehat{\sigma}_{j}}^{(\nu)} \psi_{j}\left(\widehat{t}_{m_{j}}\right), \quad \psi_{j}\left(\widehat{x}_{j}\right)=\int_{\mathbb{R}} g_{j}(x) d x_{j} .
$$

Moreover, by [11, (4.11)],

$$
\mathcal{R}_{\sigma} f_{j, h}(t) \leq c h^{r_{j}-\delta} t_{m_{j}}^{\delta-1} F_{j}\left(\widehat{t}_{m_{j}}\right)
$$

By (2.3) we have

$$
\left\|F_{j}\right\|_{1, \mathbb{R}_{+}^{n-1}} \leq c\left\|\psi_{j}\right\|_{1, \mathbb{R}^{n-1}}=\left\|g_{j}\right\|_{1} .
$$

So, for any $l \neq m_{j}, l \in\{1, \ldots, n\}$ and $0<\delta<1$ we apply Lemma 1 to $F_{j}$ and obtain a function $\phi\left(t_{l}\right)$ satisfying (3.5). Furthermore,

$$
F_{j}\left(\widehat{t}_{m_{j}}\right) \leq c \pi\left(\widehat{t}_{m_{j}}\right)^{-1} \phi\left(t_{l}\right) \text {. }
$$

Thus, by (3.11) and (3.12) we get (3.6) and (3.7). Finally,

$$
\|\phi\|_{\mathcal{L}} \leq c\left\|F_{j}\right\|_{1}
$$

and (3.13) imply (3.4).

Lemma 3. Let $n \in \mathbb{N}, j \in\{1, \ldots, n\}, 0<r_{j}<\infty$ and $1 \leq p<\infty$. Let $f \in L^{p}\left(\mathbb{R}^{n}\right)$. Then, for any $\sigma \in \mathcal{P}_{n}$ and any $K>1$,

$$
\mathcal{R}_{\sigma} f(t) \leq 2^{\bar{r}_{j}} \mathcal{R}_{\sigma_{j}^{\prime}} f\left(K t_{m_{j}}, \widehat{t}_{m_{j}} / 2\right)+c(K) \pi(t)^{-1 / p} \omega_{j}^{\bar{r}_{j}}\left(f ; t_{m_{j}}\right)_{p}
$$

and

$$
\mathcal{R}_{\sigma} f_{j, h}(t) \leq \pi(t)^{-1 / p} \omega_{j}^{\bar{r}_{j}}(f ; h)_{p},
$$

where $\sigma_{j}^{\prime}$ is obtained from $\sigma$ by moving the $j$ th index to the first position, and $m_{j}=\sigma^{-1}(j)$.

Proof. By [11, (3.3)], for any $K>1$ we have

$$
\mathcal{R}_{\sigma} f(t) \leq 2^{\bar{r}_{j}} \mathcal{R}_{\sigma_{j}^{\prime}} f\left(K t_{m_{j}}, \widehat{t}_{m_{j}} / 2\right)+\mathcal{R}_{\sigma} \Phi_{j}(t / 2),
$$

where

$$
\Phi_{j}(x)=\frac{1}{t_{m_{j}}} \int_{0}^{\left(\bar{r}_{j}+1\right) K t_{m_{j}}}\left|\Delta_{j}^{\bar{r}_{j}}(h) f(x)\right| d h .
$$

Moreover, by (2.1),

$$
\mathcal{R}_{\sigma} \Phi_{j}(t / 2) \leq \Phi_{j}^{*}\left(\pi(t) / 2^{n}\right) .
$$

We choose a measurable set $E \subset \mathbb{R}^{n}$ such that $|E| \geq \pi(t) / 2^{n}$ and $\left|\Phi_{j}(x)\right| \geq$ $\Phi_{j}^{*}\left(\pi(t) / 2^{n}\right)$ for all $x \in E$. Integrating over $E$, applying the Fubini theorem 
and using Hölder's inequality, we get

$$
\begin{aligned}
\Phi_{j}^{*}\left(\frac{\pi(t)}{2^{n}}\right) & \leq \frac{1}{|E|} \int_{E} \Phi_{j}(x) d x \\
& =\frac{1}{|E| t_{m_{j}}} \int_{0}^{\left(\bar{r}_{j}+1\right) K t_{m_{j}}}\left(\int_{E}\left|\Delta_{j}^{\bar{r}_{j}}(h) f(x)\right| d x\right) d h \\
& \leq \frac{1}{|E|^{1 / p} t_{m_{j}}} \int_{0}^{\left(\bar{r}_{j}+1\right) K t_{m_{j}}}\left\|\Delta_{j}^{\bar{r}_{j}}(h) f\right\|_{p} d h \\
& \leq c(K) \pi(t)^{-1 / p} \omega_{j}^{\bar{r}_{j}}\left(f ; t_{m_{j}}\right)_{p} .
\end{aligned}
$$

Now (3.16)-(3.18) imply (3.14). Inequality (3.15) is immediate; indeed, we have

$$
\mathcal{R}_{\sigma} f_{j, h}(t) \leq f_{j, h}^{*}(\pi(t)) \leq \pi(t)^{-1 / p}\left\|f_{j, h}\right\|_{p} \leq \pi(t)^{-1 / p} \omega_{j}^{\bar{r}_{j}}(f ; h)_{p} .
$$

REMARK 1. If $f \in\left[\bigcap_{j: r_{j} \in \mathbb{N}} W_{p ; j}^{r_{j}}\left(\mathbb{R}^{n}\right)\right] \cap\left[\bigcap_{j: r_{j} \notin \mathbb{N}} H_{p ; j}^{r_{j}}\left(\mathbb{R}^{n}\right)\right]$, then we can simultaneously apply the estimates obtained in Lemmas 2 and 3. Let $\sigma \in \mathcal{P}_{n}$, $K>1$, and $0<\delta<1$. If $r_{j} \in \mathbb{N}$, choose $l_{j} \neq \sigma^{-1}(j)$ and denote by $\phi_{j}$ the function $\phi \equiv \phi_{j, l_{j}, \sigma, \delta}$ defined in Lemma 2. If $r_{j} \notin \mathbb{N}$ define $\Omega_{j} \equiv$ $\sup _{u>0} u^{-r_{j}} \omega_{j}^{\bar{r}_{j}}(f ; u)_{p}$. Now, combining (3.6) and (3.14) gives

$$
\mathcal{R}_{\sigma} f(t) \leq 2^{\bar{r}^{\prime}} \sum_{j=1}^{n} \mathcal{R}_{\sigma_{j}^{\prime}} f\left(K t_{m_{j}}, \widehat{t}_{m_{j}} / 2\right)+c(K) \pi(t)^{-1 / p} \varrho_{\sigma}(t)
$$

where $m_{j}=\sigma^{-1}(j), \bar{r}^{\prime} \equiv \max \bar{r}_{j}$ and

$$
\varrho_{\sigma}(t)=\min \left\{\min _{r_{j} \in \mathbb{N}}\left\{t_{m_{j}}^{r_{j}} \phi_{j}\left(t_{l_{j}}\right)\right\}, \min _{r_{j} \notin \mathbb{N}}\left\{t_{m_{j}}^{r_{j}} \Omega_{j}\right\}\right\} \text {. }
$$

4. The main lemma. In this section we prove the main lemmas that form the base of our approach (see Lemmas 5, 7, and 8 below). It will be convenient to use the following auxiliary proposition.

Lemma 4. Let $m \in \mathbb{N}$ and $0<\alpha_{i}<\infty(i=1, \ldots, m)$. Define $\alpha=$ $\left(\sum_{i=1}^{m} \alpha_{i}^{-1}\right)^{-1}$. Let $a, b>0$ be such that $a / b<\alpha$. Set

$$
\varrho(z)=\min \left\{\lambda, z_{1}^{\alpha_{1}} \lambda_{1}, \ldots, z_{m}^{\alpha_{m}} \lambda_{m}\right\} \quad\left(z \in \mathbb{R}_{+}^{m}\right)
$$

where $\lambda, \lambda_{1}, \ldots, \lambda_{m}$ are positive constants. Then

$$
\int_{\mathbb{R}_{+}^{m}} \varrho(z)^{b} \pi(z)^{-a} \frac{d z}{\pi(z)} \leq c \lambda^{b-a / \alpha} \prod_{i=1}^{m} \lambda_{i}^{a / \alpha_{i}}
$$

where $c$ is a constant that only depends on $\alpha_{i}, a, b$. 
Proof. Set $\varrho_{i}\left(z_{i}\right)=\min \left\{\lambda, z_{i}^{\alpha_{i}} \lambda_{i}\right\}, i=1, \ldots, m$. Denote by $I$ the left hand side of (4.1). It is clear that

$$
I \leq \int_{\mathbb{R}_{+}^{m}} \prod_{i=1}^{m} \varrho_{i}\left(z_{i}\right)^{b \alpha / \alpha_{i}} z_{i}^{-a} \frac{d z}{\pi(z)}=\prod_{i=1}^{m} I_{i},
$$

where

$$
I_{i}=\int_{0}^{\infty} \varrho_{i}\left(z_{i}\right)^{b \alpha / \alpha_{i}} z_{i}^{-a} \frac{d z_{i}}{z_{i}} .
$$

Now,

$$
\begin{aligned}
I_{i} & =\lambda_{i}^{b \alpha / \alpha_{i}} \int_{0}^{\left(\lambda / \lambda_{i}\right)^{1 / \alpha_{i}}} z_{i}^{b \alpha-a} \frac{d z_{i}}{z_{i}}+\lambda^{b \alpha / \alpha_{i}} \int_{\left(\lambda / \lambda_{i}\right)^{1 / \alpha_{i}}}^{\infty} z_{i}^{-a} \frac{d z_{i}}{z_{i}} \\
& =c\left[\lambda^{b \alpha-a} \lambda_{i}^{a}\right]^{1 / \alpha_{i}} .
\end{aligned}
$$

By (4.2) and (4.3), we immediately obtain (4.1).

From now on, let $n \in \mathbb{N}$, and $0<r_{i}<\infty$, and $1 \leq \theta_{i} \leq \infty$. Assume that $\phi_{i} \in \mathcal{L}^{\theta_{i}}$ are positive functions $(i=1, \ldots, n)$. Define

$$
\varrho(t)=\min \left\{t_{1}^{r_{1}} \phi_{1}\left(t_{l_{1}}\right), t_{2}^{r_{2}} \phi_{2}\left(t_{l_{2}}\right), \ldots, t_{n}^{r_{n}} \phi_{n}\left(t_{l_{n}}\right)\right\},
$$

where $l_{1}, \ldots, l_{n} \in\{1, \ldots, n\}$.

REMARK 2. Note that the function $\varrho_{\sigma}(t)$ defined in (3.20) is a particular case of (4.4) $\left(\theta_{i}=p\right.$ if $r_{i} \in \mathbb{N}$, and $\theta_{i}=\infty, \phi_{i} \equiv \Omega_{i}$ if $\left.r_{i} \notin \mathbb{N}\right)$.

The lemma below gives us the integrability for functions of type (4.4).

Lemma 5 (The Main Lemma). Let $n \in \mathbb{N}, 0<r_{i}<\infty$, and $1 \leq \theta_{i} \leq \infty$ $(i=1, \ldots, n)$. Let $\varrho(t)=\min _{1 \leq i \leq n}\left\{t_{i}^{r_{i}} \phi_{i}\left(t_{l_{i}}\right)\right\}$, where $\phi_{i} \in \mathcal{L}^{\theta_{i}}$ and $\bar{l}_{i} \in$ $\{1, \ldots, n\}$. Set

$$
r=n\left(\sum_{i=1}^{n} \frac{1}{r_{i}}\right)^{-1}, \quad s=\frac{n}{r}\left(\sum_{i=1}^{n} \frac{1}{r_{i} \theta_{i}}\right)^{-1} .
$$

Then

$$
\left(\int_{\mathbb{R}_{+}^{n}} \varrho(t)^{s} \pi(t)^{-r s / n} \frac{d t}{\pi(t)}\right)^{1 / s} \leq c \sum_{i=1}^{n}\left\|\phi_{i}\right\|_{\mathcal{L}^{\theta_{i}}},
$$

where $c$ is a finite constant that only depends on $n, r_{i}, \theta_{i}$.

Proof. We can assume that

$$
\sum_{i=1}^{n}\left\|\phi_{i}\right\|_{\mathcal{L}^{\theta_{i}}}=1 .
$$

Moreover, we can suppose that not all $\theta_{i}$ 's are equal to infinity $\left({ }^{1}\right)$.

$\left({ }^{1}\right)$ Otherwise $s=\infty$ and the result is trivial. 
Define $\phi(u)=\sum_{i=1, \ldots, n ; \theta_{i} \neq \infty} \phi_{i}(u)^{\theta_{i}}(u>0)$. By $(4.7),\|\phi\|_{\mathcal{L}^{1}} \leq 1$. Set

$$
B_{k}=\left\{t \in \mathbb{R}_{+}^{n}: \max _{i=1, \ldots, n ; \theta_{i} \neq \infty} \phi\left(t_{l_{i}}\right) \leq \phi\left(t_{k}\right)\right\} .
$$

It is clear that $\bigcup_{k=1}^{n} B_{k}=\mathbb{R}_{+}^{n}$.

Without loss of generality we consider the integral of the left side of (4.6) only over $B_{1}$. For almost all $t \in B_{1}$ we get

$$
\varrho(t) \leq \varrho_{B_{1}}(t) \equiv \min \left\{t_{1}^{r_{1}} \phi\left(t_{1}\right)^{1 / \theta_{1}}, \ldots, t_{n}^{r_{n}} \phi\left(t_{1}\right)^{1 / \theta_{n}}\right\} .
$$

Hence

$$
\begin{aligned}
\int_{B_{1}} \varrho(t)^{s} \pi(t)^{-r s / n} & \frac{d t}{\pi(t)} \leq \int_{\mathbb{R}_{+}^{n}} \varrho_{B_{1}}(t)^{s} \pi(t)^{-r s / n} \frac{d t}{\pi(t)} \\
& =\int_{0}^{\infty} t_{1}^{-r s / n} \frac{d t_{1}}{t_{1}} \int_{\mathbb{R}_{+}^{n-1}} \varrho_{B_{1}}\left(t_{1}, \widehat{t}_{1}\right)^{s} \pi\left(\widehat{t}_{1}\right)^{-r s / n} \frac{d \widehat{t}_{1}}{\pi\left(\widehat{t}_{1}\right)}
\end{aligned}
$$

For each fixed $t_{1} \in \mathbb{R}_{+}$, applying Lemma 4 and (4.5), we get

$$
\begin{aligned}
& \int_{\mathbb{R}_{+}^{n-1}} \varrho_{B_{1}}\left(t_{1}, \widehat{t}_{1}\right)^{s} \pi\left(\widehat{t}_{1}\right)^{-r s / n} d \widehat{t}_{1} \\
& \quad \leq c\left[t_{1}^{r_{1}} \phi\left(t_{1}\right)^{1 / \theta_{1}}\right]^{s-\sum_{i=2}^{n} \frac{r s}{n r_{i}}} \phi\left(t_{1}\right)^{\sum_{i=2}^{n} \frac{r s}{n \theta_{i} r_{i}}}=c \phi\left(t_{1}\right) t_{1}^{r s / n} .
\end{aligned}
$$

Since $\|\phi\|_{\mathcal{L}^{1}} \leq 1$, (4.9) and (4.10) yield

$$
\int_{B_{1}} \varrho(t)^{s} \pi(t)^{-r s / n} \frac{d t}{\pi(t)} \leq c .
$$

We will obtain a generalization of Lemma 5. For this purpose, we need the following Hardy type inequality.

Lemma 6. Let $\varphi$ be a measurable non-negative function on $\mathbb{R}_{+}$. Let $\delta, \alpha$ $>0$ and let $1 \leq \gamma<\infty$. Assume that $\beta$ is a positive measurable function on $\mathbb{R}_{+}$such that $\beta(u) u^{-\delta}$ increases. Then

$$
\int_{0}^{\infty} h^{-\alpha-1} d h\left(\int_{\{h \geq \beta(u)\}} \varphi(u) \frac{d u}{u}\right)^{\gamma} \leq c \int_{0}^{\infty} \beta(u)^{-\alpha} \varphi(u)^{\gamma} \frac{d u}{u}
$$

and

$$
\int_{0}^{\infty} h^{\alpha-1} d h\left(\int_{\{h \leq \beta(u)\}} \varphi(u) \frac{d u}{u}\right)^{\gamma} \leq c \int_{0}^{\infty} \beta(u)^{\alpha} \varphi(u)^{\gamma} \frac{d u}{u},
$$

where $c$ is a constant that only depends on $\alpha, \delta$ and $\gamma$.

Proof. As $\beta(u) u^{-\delta} \uparrow$, the inverse function $\beta^{-1}$ exists on $\mathbb{R}_{+}$and satisfies the condition

$$
\beta^{-1}(2 u) \leq 2^{1 / \delta} \beta^{-1}(u)
$$


Denote by $I$ the left hand side of (4.11). We have

$$
\begin{aligned}
I & \equiv \int_{0}^{\infty} h^{-\alpha-1} d h\left(\int_{0}^{\beta^{-1}(h)} \varphi(u) \frac{d u}{u}\right)^{\gamma} \\
& =\int_{0}^{\infty} h^{-\alpha-1} d h\left(\sum_{k=0}^{\infty} \int_{\beta^{-1}\left(2^{-k-1} h\right)}^{\beta^{-1}\left(2^{-k} h\right)} \varphi(u) \frac{d u}{u}\right)^{\gamma} .
\end{aligned}
$$

Next, by Minkowski's inequality,

$$
\begin{aligned}
I^{1 / \gamma} & \leq \sum_{k=0}^{\infty}\left(\int_{0}^{\infty} h^{-\alpha-1} d h\left(\int_{\beta^{-1}\left(2^{-k-1} h\right)}^{\beta^{-1}\left(2^{-k} h\right)} \varphi(u) \frac{d u}{u}\right)^{\gamma}\right)^{1 / \gamma} \\
& =\sum_{k=0}^{\infty} 2^{-k \alpha / \gamma}\left(\int_{0}^{\infty} z^{-\alpha-1} d z\left(\int_{\beta^{-1}(z / 2)}^{\beta^{-1}(z)} \varphi(u) \frac{d u}{u}\right)^{\gamma}\right)^{1 / \gamma}
\end{aligned}
$$

Further, using Hölder's inequality and (4.13) we obtain

$$
\int_{\beta^{-1}(z / 2)}^{\beta^{-1}(z)} \varphi(u) \frac{d u}{u} \leq c\left(\int_{0}^{\beta^{-1}(z)} \varphi(u)^{\gamma} \frac{d u}{u}\right)^{1 / \gamma}
$$

Thus, by Fubini's theorem,

$$
I \leq c \int_{0}^{\infty} z^{-\alpha-1} d z \int_{0}^{\beta^{-1}(z)} \varphi(u)^{\gamma} \frac{d u}{u}=c \int_{0}^{\infty} \beta(u)^{-\alpha} \varphi(u)^{\gamma} \frac{d u}{u} .
$$

The same reasoning proves (4.12).

LEMMA 7. Assume that the conditions of Lemma 5 hold and suppose that there exists $0<\delta \leq \frac{1}{2} \min _{1 \leq i, k \leq n, \theta_{k} \neq \infty}\left\{r_{i} / \theta_{k}\right\}$ such that

$$
\phi_{i}(u) u^{\delta} \uparrow \quad \text { and } \quad \phi_{i}(u) u^{-\delta} \downarrow
$$

for every $i$ such that $\theta_{i}<\infty$. Then, for any $0<d \leq \infty$ and $j \in\{1, \ldots, n\}$,

$$
\left(\int_{0}^{\infty}\left\|\varrho(t)^{s} \pi(t)^{-r s / n}\right\|_{L^{d}\left(\mathbb{R}_{+}^{n-1}, d \widehat{t}_{j} / \pi\left(\widehat{t}_{j}\right)\right)} \frac{d t_{j}}{t_{j}}\right)^{1 / s} \leq c \sum_{i=1}^{n}\left\|\phi_{i}\right\|_{\mathcal{L}^{\theta_{i}}}
$$

where $c$ is a constant that depends on $n, r_{i}, \theta_{i}, d, \delta$.

Note that the greater is $d$, the weaker is (4.15). Indeed, by (4.14), the function $\varrho(t) \pi(t)^{\delta}$ is increasing in each variable. So, it is easy to see that

$$
\sup _{\widehat{t}_{j} \in \mathbb{R}_{+}^{n-1}} \varrho(t)^{s} \pi(t)^{-r s / n} \leq c\left\|\varrho(t)^{s} \pi(t)^{-r s / n}\right\|_{L^{d}\left(\mathbb{R}_{+}^{n-1}, d \widehat{t}_{j} / \pi\left(\widehat{t}_{j}\right)\right)}
$$

$$
\text { for any } 0<d<\infty \text {. }
$$


It follows that if $q>d>0$, then

$$
\begin{aligned}
& \left\|\varrho(t)^{s} \pi(t)^{-r s / n}\right\|_{L^{q}\left(\mathbb{R}_{+}^{n-1}, d \widehat{t}_{j} / \pi\left(\widehat{t}_{j}\right)\right)} \\
& \leq\left[\left\|\varrho(t)^{s} \pi(t)^{-r s / n}\right\|_{L^{d}\left(\mathbb{R}_{+}^{n-1}, d \widehat{t}_{j} / \pi\left(\widehat{t}_{j}\right)\right)}\right]^{(q-d) / q}\left(\int_{\mathbb{R}_{+}^{n-1}}\left[\varrho(t)^{s} \pi(t)^{-r s / n}\right]^{d} \frac{d \widehat{t}_{j}}{\pi\left(\widehat{t}_{j}\right)}\right)^{1 / q} \\
& \leq c\left\|\varrho(t)^{s} \pi(t)^{-r s / n}\right\|_{L^{d}\left(\mathbb{R}_{+}^{n-1}, d \widehat{t}_{j} / \pi\left(\widehat{t}_{j}\right)\right)}
\end{aligned}
$$

Note also that for $d=1$ we get the same conclusion as in Lemma 5. So, for the proof, we can suppose that $0<d<1$.

Proof of Lemma 7. As above, we can suppose that condition (4.7) holds. Let $\phi$ and $B_{k}(k=1, \ldots, n)$ be as defined in the proof of Lemma 5 . Then the left hand side of (4.15) does not exceed $\sum_{k=1}^{n} I_{k}$, where

$$
I_{k}=\left(\int_{0}^{\infty}\left\|\varrho(t)^{s} \pi(t)^{-r s / n} \chi_{B_{k}}(t)\right\|_{L^{d}\left(\mathbb{R}_{+}^{n-1}, d \widehat{t}_{j} / \pi\left(\widehat{t}_{j}\right)\right)} \frac{d t_{j}}{t_{j}}\right)^{1 / s} .
$$

We consider $I_{1}$. For almost all $t \in B_{1}$ we have the inequality (4.8). Thus,

$$
I_{1}^{s} \leq \int_{0}^{\infty}\left\|\varrho_{B_{1}}(t)^{s} \pi(t)^{-r s / n}\right\|_{L^{d}\left(\mathbb{R}_{+}^{n-1}, d \widehat{t}_{j} / \pi\left(\widehat{t}_{j}\right)\right)} \frac{d t_{j}}{t_{j}}
$$

CAsE 1: $j=1$. Then

$$
I_{1}^{s} \leq \int_{0}^{\infty} t_{1}^{-r s / n} G\left(t_{1}\right) \frac{d t_{1}}{t_{1}}
$$

where

$$
G\left(t_{1}\right)^{d} \equiv \int_{\mathbb{R}_{+}^{n-1}}\left[\varrho_{B_{1}}(t)^{s} \pi\left(\widehat{t}_{1}\right)^{-r s / n}\right]^{d} \frac{d \widehat{t_{1}}}{\pi\left(\widehat{t_{1}}\right)}
$$

Applying Lemma 4 to the variables $t_{2}, \ldots, t_{n}$, we easily get

$$
G\left(t_{1}\right)^{d} \leq c\left[t_{1}^{r_{1}} \phi\left(t_{1}\right)^{1 / \theta_{1}}\right]^{\frac{r s d}{n r_{1}}} \phi\left(t_{1}\right)^{d \sum_{i=2}^{n} \frac{r s}{n r_{i} \theta_{i}}}=\left[t_{1}^{r s / n} \phi\left(t_{1}\right)\right]^{d} .
$$

This implies (4.15).

CAsE $2: j \neq 1$. For $t \in \mathbb{R}_{+}^{n}$, denote by $\widehat{t}_{1, j}$ the $(n-2)$-dimensional vector obtained from $t$ by removal of $t_{1}, t_{j}$. Then

$$
I_{1}^{s} \leq \int_{0}^{\infty} t_{j}^{-r s / n}\left\|R\left(t_{1}, t_{j}\right)\right\|_{L^{d}\left(\mathbb{R}_{+}, d t_{1} / t_{1}\right)} \frac{d t_{j}}{t_{j}}
$$

where

$$
R\left(t_{1}, t_{j}\right)^{d}=t_{1}^{-r s d / n} \int_{\mathbb{R}_{+}^{n-2}}\left[\pi\left(\widehat{t}_{1, j}\right)^{-r s / n} \varrho_{B_{1}}(t)^{s}\right]^{d} \frac{d \widehat{t}_{1, j}}{\pi\left(\widehat{t}_{1, j}\right)}
$$


Fix $t_{1}, t_{j}$ and apply Lemma 4 to the coordinates of the vector $\widehat{t}_{1, j}$. We obtain $R\left(t_{1}, t_{j}\right) \leq c t_{1}^{-r s / n} \min \left\{t_{1}^{r_{1}} \phi\left(t_{1}\right)^{1 / \theta_{1}}, t_{j}^{r_{j}} \phi\left(t_{1}\right)^{1 / \theta_{j}}\right\}^{\frac{r s}{n}\left(\frac{1}{r_{1}}+\frac{1}{r_{j}}\right)} \phi\left(t_{1}\right)^{\sum_{i \neq 1, j} \frac{r s}{n r_{i} \theta_{i}}}$.

Now, we define $\beta\left(t_{1}\right)=\left[t_{1}^{r_{1}} \phi\left(t_{1}\right)^{1 / \theta_{1}-1 / \theta_{j}}\right]^{1 / r_{j}}$. Note that by (4.14),

$$
\beta\left(t_{1}\right) t_{1}^{-\delta / r_{j}} \uparrow \text {. }
$$

Moreover $\left(b \equiv \frac{r s}{n r_{j} \theta_{1}}+\sum_{i \neq j} \frac{r s}{n r_{i} \theta_{i}}, b^{\prime} \equiv \frac{r s}{n r_{1} \theta_{j}}+\sum_{i \neq 1} \frac{r s}{n r_{i} \theta_{i}}\right)$,

$$
\leq c \begin{cases}t_{1}^{\frac{r s}{n} \frac{r_{1}}{r_{j}}} \phi\left(t_{1}\right)^{b} \equiv R_{1}\left(t_{1}\right) & \text { if } \beta\left(t_{1}\right) \leq t_{j}, \\ t_{j}^{\frac{r s}{n}\left(1+\frac{r_{j}}{r_{1}}\right)} t_{1}^{-\frac{r s}{n}} \phi\left(t_{1}\right)^{b^{\prime}} \equiv t_{j}^{\frac{r s}{n}\left(1+\frac{r_{j}}{r_{1}}\right)} R_{2}\left(t_{1}\right) & \text { if } \beta\left(t_{1}\right) \geq t_{j} .\end{cases}
$$

Combining (4.16) and (4.18), we get

$$
\begin{aligned}
I_{1}^{s} \leq & c \int_{0}^{\infty} t_{j}^{-\frac{r s}{n}} \frac{d t_{j}}{t_{j}}\left(\int_{\left\{t_{j} \geq \beta\left(t_{1}\right)\right\}} R_{1}\left(t_{1}\right)^{d} \frac{d t_{1}}{t_{1}}\right)^{1 / d} \\
& +c \int_{0}^{\infty} t_{j}^{\frac{r s}{n}} \frac{r_{j}}{r_{1}} \frac{d t_{j}}{t_{j}}\left(\int_{\left\{t_{j} \leq \beta\left(t_{1}\right)\right\}} R_{2}\left(t_{1}\right)^{d} \frac{d t_{1}}{t_{1}}\right)^{1 / d} .
\end{aligned}
$$

Taking into account (4.17), we apply Lemma 6 with $\gamma=1 / d(\gamma>1)$. Using the definitions of $\beta, R_{1}$, and $R_{2}$, and (4.5), we obtain

$$
I_{1}^{s} \leq c \int_{0}^{\infty} \beta\left(t_{1}\right)^{-\frac{r s}{n}} R_{1}\left(t_{1}\right) \frac{d t_{1}}{t_{1}}+c \int_{0}^{\infty} \beta\left(t_{1}\right)^{\frac{r s}{n} \frac{r_{j}}{r_{1}}} R_{2}\left(t_{1}\right) \frac{d t_{1}}{t_{1}}=c^{\prime} \int_{0}^{\infty} \phi\left(t_{1}\right) \frac{d t_{1}}{t_{1}} \text {. }
$$

We will also use the following generalization of Lemma 7 .

Lemma 8. Let $m \in \mathbb{N}, 0<r_{i}<\infty$, and $1 \leq \theta_{i} \leq \infty(i=1, \ldots, m)$. Define the function $\varrho(z)=\min _{1 \leq i \leq m}\left\{z_{i}^{r_{i}} \phi_{i}\left(z_{l_{i}}\right)\right\}$, where $\phi_{i} \in \mathcal{L}^{\theta_{i}}$ and $l_{i} \in$ $\{1, \ldots, m\}$. Suppose also that there exists $0<\delta \leq \frac{1}{2} \min _{1 \leq i, k \leq m, \theta_{k} \neq \infty}\left\{r_{i} / \theta_{k}\right\}$ such that

$$
\phi_{i}(u) u^{\delta} \uparrow \quad \text { and } \quad \phi_{i}(u) u^{-\delta} \downarrow
$$

for every $i$ such that $\theta_{i}<\infty$. Let $0<a_{i}<\infty$ be numbers satisfying

$$
\sum_{i=1}^{m} \frac{a_{i}}{r_{i} \theta_{i}}=1
$$

Set $a \equiv \sum_{i=1}^{m} a_{i} / r_{i}$. Then, for any $0<d \leq \infty$ and $j \in\{1, \ldots, m\}$,

$$
\left(\int_{0}^{\infty}\left\|\varrho(z)^{a} \prod_{i=1}^{m} z_{i}^{-a_{i}}\right\|_{L^{d}\left(\mathbb{R}_{+}^{m-1}, d \widehat{z}_{j} / \pi\left(\widehat{z}_{j}\right)\right)} \frac{d z_{j}}{z_{j}}\right)^{1 / a} \leq c \sum_{i=1}^{m}\left\|\phi_{i}\right\|_{\mathcal{L}^{\theta_{i}}},
$$

where $c$ is a constant that depends on $m, r_{i}, a_{i}, \theta_{i}, d, \delta$.

Note that Lemma 7 is the particular case $a_{1}=\cdots=a_{m}$. 
Proof. Let $J$ be the left hand side of (4.20). The change of variable $z_{i}^{a_{i} / b}=u_{i}\left(b=\min _{1 \leq k \leq m} a_{k}\right)$ gives us

$$
J=c\left(\int_{0}^{\infty}\left\|\varrho(u)^{a} \pi(u)^{-b}\right\|_{L^{d}\left(\mathbb{R}_{+}^{m-1}, d \widehat{u}_{j} / \pi\left(\widehat{u}_{j}\right)\right)} \frac{d u_{j}}{u_{j}}\right)^{1 / a},
$$

where $\varrho(u)=\min _{1 \leq i \leq m}\left\{u_{i}^{r_{i}^{\prime}} F_{i}\left(u_{l_{i}}\right)\right\}, r_{i}^{\prime}=r_{i} b / a_{i}$, and $F_{i}(v)=\phi_{i}\left(v^{b / a_{l_{i}}}\right)$ belongs to $\mathcal{L}^{\theta_{i}}$. Note now that $F_{i}(v) v^{\delta} \uparrow$ and $F_{i}(v) v^{-\delta} \downarrow$. So, it remains to apply Lemma 7 to the last integral $\left(r^{\prime}=m\left(\sum 1 / r_{i}^{\prime}\right)^{-1}=a^{-1} b m\right.$ and $r^{\prime} s^{\prime} / m=\left(\sum 1 / r_{i}^{\prime} \theta_{i}\right)^{-1}=b$ by (4.19)) and we get (4.20).

\section{Embeddings of Lipschitz spaces} Set

Theorem 1. Let $2 \leq n \in \mathbb{N}, 1 \leq p<\infty$, and $0<r_{i}<\infty(i=1, \ldots, n)$.

$$
r=n\left(\sum_{i=1}^{n} \frac{1}{r_{i}}\right)^{-1}, \quad r^{\prime}=n\left(\sum_{i: r_{i} \in \mathbb{N}} \frac{1}{r_{i}}\right)^{-1}, \quad s=\frac{r^{\prime} p}{r}, \quad q^{*}=\frac{n p}{n-r p} .
$$

If $p<n / r$, then

$$
\|f\|_{q^{*}, s ; \mathcal{R}} \leq c\|f\|_{\lambda_{p}^{r_{1}, \ldots, r_{n}}} \quad \text { for all } f \in \Lambda_{p}^{r_{1}, \ldots, r_{n}}\left(\mathbb{R}^{n}\right),
$$

where $c$ is a constant that does not depend on $f$.

Proof. First suppose that $f \in C_{0}^{\infty}\left(\mathbb{R}^{n}\right)$. Let $S=\|f\|_{q^{*}, s ; \mathcal{R}}$. So, $S<\infty$.

It is well known that if $1<p \leq \infty$, then

$$
\|f\|_{\lambda_{p}^{r_{1}, \ldots, r_{n}}} \sim \sum_{j: r_{j} \in \mathbb{N}}\left\|D_{j}^{r_{j}} f\right\|_{p}+\sum_{j: r_{j} \notin \mathbb{N}} \sup _{u>0} u^{-r_{j}} \omega_{j}^{\bar{r}_{j}}(f ; u)_{p},
$$

and this is still true for $p=1$ when restricted to functions in $C^{\infty}\left(\mathbb{R}^{n}\right)$ [10].

Now, taking into account (5.3) and Remark 1, we integrate inequality (3.19) and get, for any $\sigma \in \mathcal{P}_{n}$,

$$
\begin{aligned}
& \left(\int_{\mathbb{R}_{+}^{n}} \pi(t)^{s / q^{*}-1} \mathcal{R}_{\sigma} f(t)^{s} d t\right)^{1 / s} \\
& \quad \leq 2^{\bar{r}^{\prime}+n} K^{-1 / q^{*}} S+c(K)\left(\int_{\mathbb{R}_{+}^{n}} \pi(t)^{-r s / n-1} \varrho_{\sigma}(t)^{s} d t\right)^{1 / s}
\end{aligned}
$$

with $\varrho_{\sigma}(t)$ defined in (3.20). Consequently,

$$
\begin{aligned}
S=\sum_{\sigma \in \mathcal{P}_{n}}\|f\|_{q^{*}, s ; \mathcal{R}_{\sigma}} \leq & n ! 2^{\bar{r}^{\prime}+n} K^{-1 / q^{*}} S \\
& +c^{\prime}(K) \sum_{\sigma \in \mathcal{P}_{n}}\left(\int_{\mathbb{R}_{+}^{n}} \pi(t)^{-r s / n-1} \varrho_{\sigma}(t)^{s} d t\right)^{1 / s} .
\end{aligned}
$$


Now, we apply Lemma 5 with $\theta_{i}=p$ if $r_{i} \in \mathbb{N}$, and $\theta_{i}=\infty$ and $\phi_{i}=\Omega_{i}$ if $r_{i} \notin \mathbb{N}$ (observe that the values of $s$ in (5.1) and (4.5) coincide), to get

$$
\left(\int_{\mathbb{R}_{+}^{n}} \pi(t)^{-r s / n-1} \varrho_{\sigma}(t)^{s} d t\right)^{1 / s} \leq c\left[\sum_{r_{i} \in \mathbb{N}}\left\|\phi_{i}\right\|_{\mathcal{L}^{p}}+\sum_{r_{i} \notin \mathbb{N}} \Omega_{i}\right] .
$$

Therefore, setting $K=\left(2^{\bar{r}^{\prime}+n+1} n !\right)^{q^{*}}$, and using (5.4), (5.5), the definition of $\Omega_{i}$, and (3.4) we obtain the inequality (5.2).

For $f \in \Lambda_{p}^{r_{1}, \ldots, r_{n}}\left(\mathbb{R}^{n}\right)$, there exists a sequence of functions $f_{k} \in C_{0}^{\infty}\left(\mathbb{R}^{n}\right)$ such that $\lim _{k \rightarrow \infty}\left\|f_{k}\right\|_{\lambda_{p}^{r_{1}, \ldots, r_{n}}} \leq\|f\|_{\lambda_{p}^{r_{1}, \ldots, r_{n}}}$ and $f_{k} \rightarrow f$ in $L^{p}$ (use $\varepsilon$ regularizations and cut-off). So, applying Lemma 2 of [11] and Fatou's Lemma we obtain (5.2) in the general case.

REMARK 3. If all the $r_{i}$ 's are integers, then $s=p$ and we get the embedding of anisotropic Sobolev spaces into Lorentz spaces proved earlier in $[9,11]$. In the general case, assume that $s \leq q^{*}$. Then Theorem 1 yields an alternative proof of the results concerning embeddings into $L^{q^{*}}$ (see [5]) and $L^{q^{*}, s}($ see $[14,10])$.

Theorem 2. Let $2 \leq n \in \mathbb{N}, 1 \leq p<q<\infty$, and $0<r_{i}<\infty$ $(i=1, \ldots, n)$. Define $r, s$ as in (5.1). Suppose that

$$
\varkappa=1-\frac{n}{r}\left(\frac{1}{p}-\frac{1}{q}\right)>0
$$

and define

$$
\alpha_{i}=\varkappa r_{i}, \quad \frac{1}{\gamma_{i}}= \begin{cases}\frac{1-\varkappa}{s}+\frac{\varkappa}{p} & \text { if } r_{i} \in \mathbb{N}, \\ \frac{1-\varkappa}{s} & \text { if } r_{i} \notin \mathbb{N} .\end{cases}
$$

Then, for any $f \in \Lambda_{p}^{r_{1}, \ldots, r_{n}}\left(\mathbb{R}^{n}\right)$,

$$
\sum_{i=1}^{n}\left(\int_{0}^{\infty}\left[h^{-\alpha_{i}}\left\|\Delta_{i}^{\bar{r}_{i}}(h) f\right\|_{q, 1 ; \mathcal{R}}\right]^{\gamma_{i}} \frac{d h}{h}\right)^{1 / \gamma_{i}} \leq c\|f\|_{\lambda_{p}^{r_{1}, \ldots, r_{n}}\left(\mathbb{R}^{n}\right)}
$$

where $c$ is a constant that does not depend on $f$.

Proof. Consider the first term on the left hand side of (5.6). Define $f_{1, h}(x)=\Delta_{1}^{\bar{r}_{1}}(h) f(x)$. Then $J(h)=\left\|f_{1, h}\right\|_{q, 1 ; \mathcal{R}}<\infty$. As in Theorem 1 , we can suppose that $f \in C_{0}^{\infty}\left(\mathbb{R}^{n}\right)$.

Define now

$$
\delta \equiv \frac{1}{2} \min \left\{\frac{r}{n}(1-\varkappa), \frac{1}{p} \min _{j} r_{j}\right\} .
$$

Then, by (5.3), we proceed similarly to Remark 1. Applying Lemmas 2 and 3 
to $f_{1, h}$, we easily deduce that for any $K>1$,

$$
\mathcal{R}_{\sigma} f_{1, h}(t) \leq 2^{\bar{r}^{\prime}} \sum_{j=1}^{n} \mathcal{R}_{\sigma_{j}^{\prime}} f_{1, h}\left(K t_{m_{j}}, \widehat{t}_{m_{j}} / 2\right)+c(K) \pi(t)^{-1 / p} \varrho_{\sigma}(t),
$$

where $\varrho_{\sigma}(t)$ is defined in (3.20). Moreover, from (3.7) and (3.15) it follows that if $h<t_{m_{1}}$, then

$$
\mathcal{R}_{\sigma} f_{1, h}(t) \leq c \pi(t)^{-1 / p} \mu(t, h),
$$

where

$$
\mu(t, h)= \begin{cases}h^{r_{1}-\delta} t_{m_{1}}^{\delta} \phi_{1}\left(t_{l_{1}}\right) & \text { if } r_{1} \in \mathbb{N}, \\ h^{r_{1}} \Omega_{1} & \text { if } r_{1} \notin \mathbb{N},\end{cases}
$$

and $\phi_{1}\left(t_{l_{1}}\right)$ is defined in Lemma 2. Furthermore, setting

$$
\bar{\varrho}_{\sigma}(t, h)=\min \left\{\varrho_{\sigma}(t), \mu(t, h)\right\},
$$

for any $K>1$ we get

$$
\mathcal{R}_{\sigma} f_{1, h}(t) \leq 2^{\bar{r}^{\prime}} \sum_{j=1}^{n} \mathcal{R}_{\sigma_{j}^{\prime}} f_{1, h}\left(K t_{m_{j}}, \widehat{t}_{m_{j}} / 2\right)+c(K) \pi(t)^{-1 / p} \bar{\varrho}_{\sigma}(t, h) .
$$

Multiplying by $\pi(t)^{1 / q-1}$ and integrating over $\mathbb{R}_{+}^{n}$, we obtain

$$
\left\|f_{1, h}\right\|_{q, 1 ; \mathcal{R}_{\sigma}} \leq 2^{\bar{r}^{\prime}+n} K^{-1 / q} J(h)+c(K) J_{1}(h, \sigma),
$$

where

$$
J_{1}(h, \sigma)=\int_{\mathbb{R}_{+}^{n}} \pi(t)^{-\frac{r}{n}(1-\varkappa)} \bar{\varrho}_{\sigma}(t, h) \frac{d t}{\pi(t)} .
$$

Summing up inequalities (5.9) over all $\sigma \in \mathcal{P}_{n}$ and choosing $K=\left(2^{\bar{r}^{\prime}+n+1} n !\right)^{q}$, we get

$$
J(h) \leq c^{\prime} \sum_{\sigma \in \mathcal{P}_{n}} J_{1}(h, \sigma) .
$$

Furthermore, denoting by $I$ the first term of the left hand side of (5.6), we have (let's suppose that $\gamma_{1}<\infty\left({ }^{2}\right)$ )

$$
I \equiv\left(\int_{0}^{\infty} h^{-\alpha_{1} \gamma_{1}-1} J(h)^{\gamma_{1}} d h\right)^{1 / \gamma_{1}} \leq c^{\prime} \sum_{\sigma \in \mathcal{P}_{n}} \bar{I}(\sigma),
$$

where (by (5.11))

$$
\bar{I}(\sigma)=\left(\int_{0}^{\infty} h^{-\alpha_{1} \gamma_{1}} J_{1}(h, \sigma)^{\gamma_{1}} \frac{d h}{h}\right)^{1 / \gamma_{1}} .
$$

$\left({ }^{2}\right)$ Otherwise, none of the $r_{i}$ 's belongs to $\mathbb{N}$, and the analogue of (5.6) follows from (5.11), (5.10) and Lemma 4. 
But now, it is clear that (see (5.8), (3.20) and (5.7))

$$
\bar{\varrho}_{\sigma}(t, h) \leq\left[1+\left(t_{m_{1}} / h\right)^{\delta}\right] \widetilde{\varrho}_{\sigma}(t, h),
$$

where

$$
\widetilde{\varrho}_{\sigma}(t, h) \equiv \begin{cases}\min \left\{h^{r_{1}} \phi_{1}\left(t_{l_{1}}\right), \varrho_{\sigma}(t)\right\} & \text { if } r_{1} \in \mathbb{N}, \\ \min \left\{h^{r_{1}} \Omega_{1}, \varrho_{\sigma}(t)\right\} & \text { if } r_{1} \notin \mathbb{N} .\end{cases}
$$

So, due to (5.12), (5.10) and (5.13) we have

$$
\bar{I}(\sigma) \leq c \widetilde{I}_{1}(\sigma)+c \widetilde{I}_{2}(\sigma),
$$

where

$$
\begin{aligned}
& \widetilde{I}_{1}(\sigma) \equiv\left(\int_{0}^{\infty}\left\|h^{-\alpha_{1} \gamma_{1}} \pi(t)^{-\frac{r}{n}(1-\varkappa) \gamma_{1}} \widetilde{\varrho}_{\sigma}(t, h)^{\gamma_{1}}\right\|_{L^{1 / \gamma_{1}\left(\mathbb{R}_{+}^{n}, d t / \pi(t)\right)}} \frac{d h}{h}\right)^{1 / \gamma_{1}}, \\
& \widetilde{I}_{2}(\sigma) \\
& \quad \equiv\left(\int_{0}^{\infty}\left\|h^{-\alpha_{1} \gamma_{1}} \pi(t)^{-\frac{r}{n}(1-\varkappa) \gamma_{1}}\left(\frac{t_{m_{1}}}{h}\right)^{\delta \gamma_{1}} \widetilde{\varrho}_{\sigma}(t, h)^{\gamma_{1}}\right\|_{L^{1 / \gamma_{1}\left(\mathbb{R}_{+}^{n}, d t / \pi(t)\right)}} \frac{d h}{h}\right)^{1 / \gamma_{1}} .
\end{aligned}
$$

It remains to estimate the last two integrals.

Combining (5.14) and (3.20) we get $\widetilde{\varrho}_{\sigma}(t, h)$

$$
= \begin{cases}\min \left\{h^{r_{1}} \phi_{1}\left(t_{l_{1}}\right), \min _{r_{j} \in \mathbb{N}}\left\{t_{m_{j}}^{r_{j}} \phi_{j}\left(t_{l_{j}}\right)\right\}, \min _{r_{j} \notin \mathbb{N}}\left\{t_{m_{j}}^{r_{j}} \Omega_{j}\right\}\right\} & \text { if } r_{1} \in \mathbb{N}, \\ \min \left\{h^{r_{1}} \Omega_{1}, \min _{r_{j} \in \mathbb{N}}\left\{t_{m_{j}}^{r_{j}} \phi_{j}\left(t_{l_{j}}\right)\right\}, \min _{r_{j} \notin \mathbb{N}}\left\{t_{m_{j}}^{r_{j}} \Omega_{j}\right\}\right\} & \text { if } r_{1} \notin \mathbb{N} .\end{cases}
$$

So, $\widetilde{\varrho}_{\sigma}(t, h)$ has the form of $\varrho(z)$ in Lemma $8\left(\varrho(z)=\min _{1 \leq i \leq m}\left\{z_{i}^{r_{i}} \phi_{i}\left(z_{l_{i}}\right)\right\}\right.$, $\left.\phi_{i} \in \mathcal{L}^{\theta_{i}}\right)$. Indeed, $m=n+1, r_{n+1}=r_{1}$,

$$
z_{1}=t_{m_{1}}, \ldots, z_{n}=t_{m_{n}}, \quad z_{n+1}=h .
$$

Moreover, for $1 \leq i \leq n+1$,

$$
\left\{\begin{array}{l}
\text { If } r_{i} \in \mathbb{N} \text {, then } \theta_{i}=p \text { and } \phi_{i}(v) v^{\delta} \uparrow, \phi_{i}(v) v^{-\delta} \downarrow \quad(\text { see }(3.5)) . \\
\text { If } r_{i} \notin \mathbb{N} \text {, then } \theta_{i}=\infty \text { and } \phi_{i}=\Omega_{i} .
\end{array}\right.
$$

To estimate $\widetilde{I}_{1}(\sigma)$, note that it has the form of the left hand side of (4.20) with

$$
a_{1}=\cdots=a_{n}=\frac{r}{n}(1-\varkappa) \gamma_{1}>0, \quad a_{n+1}=\alpha_{1} \gamma_{1}>0, \quad d=1 / \gamma_{1}, \quad j=n+1 .
$$

Then $a=\gamma_{1}$ and (4.19) holds. Applying Lemma 8 we get

$$
\widetilde{I}_{1}(\sigma) \leq c\left[\sum_{r_{i} \in \mathbb{N}}\left\|\phi_{i}\right\|_{\mathcal{L}^{p}}+\sum_{r_{i} \notin \mathbb{N}} \Omega_{i}\right]
$$

And by the definition of $\Omega_{i}$ and (3.4),

$$
\widetilde{I}_{1}(\sigma) \leq c\|f\|_{\lambda_{p}^{r_{1}, \ldots, r_{n}}\left(\mathbb{R}^{n}\right)} .
$$


Next, $\widetilde{I}_{2}(\sigma)$ is also similar to the left hand side of (4.20). Indeed,

$$
\begin{gathered}
a_{n+1}=\left(\alpha_{1}+\delta\right) \gamma_{1}>0, \quad a_{1}=\left[\frac{r}{n}(1-\varkappa)-\delta\right] \gamma_{1}>0, \\
a_{i}=\frac{r}{n}(1-\varkappa) \gamma_{1}>0 \quad(i=2, \ldots, n), \quad d=1 / \gamma_{1}, \quad j=n+1 .
\end{gathered}
$$

We have $a=\gamma_{1}$ and (4.19) holds. Applying Lemma 8 we obtain the same estimate for $\widetilde{I}_{2}(\sigma)$ as for $\widetilde{I}_{1}(\sigma)$.

In addition to Theorem 1 (embedding with limit exponent) we have the following theorem.

Theorem 3. Let $2 \leq n \in \mathbb{N}, 1 \leq p<q<\infty$, and $0<r_{i}<\infty$ $(i=1, \ldots, n)$. Let $r$ be as in (5.1). Suppose that $1-(n / r)(1 / p-1 / q)>0$. Then, for any $0<\xi<\infty$,

$$
\|f\|_{q, \xi ; \mathcal{R}} \leq c\|f\|_{\Lambda_{p}^{r_{1}, \ldots, r_{n}}\left(\mathbb{R}^{n}\right)},
$$

where $c$ is a constant that does not depend on $f$.

Proof. First of all we can suppose that $f \in C_{0}^{\infty}\left(\mathbb{R}^{n}\right)$, so $S \equiv\|f\|_{q, \xi ; \mathcal{R}}$ $<\infty$. By (2.4) we can suppose that $0<\xi<1$.

By Remark 1 we get (3.19) and (3.20). For $\sigma=m^{-1} \in \mathcal{P}_{n}$ and $j=$ $1, \ldots, n$ we define

$$
\begin{aligned}
A & =\left\{t \in \mathbb{R}_{+}^{n}: t_{i} \geq 1, i=1, \ldots, n\right\}, \\
A_{\sigma, j} & =\left\{t \in \mathbb{R}_{+}^{n}: \min _{1 \leq i \leq n} t_{m_{i}}^{r_{i}}=t_{m_{j}}^{r_{j}}<1\right\} .
\end{aligned}
$$

It is clear that

$$
A \cup \bigcup_{j=1}^{n} A_{\sigma, j}=\mathbb{R}_{+}^{n}
$$

Then, by (3.19),

$$
\int_{\mathbb{R}_{+}^{n}} \pi(t)^{\xi / q-1} \mathcal{R}_{\sigma} f(t)^{\xi} d t \leq\left(2^{\bar{r}^{\prime}+n} K^{-1 / q} S\right)^{\xi}+I_{0}+c(K) \sum_{j=1}^{n} I_{j},
$$

where

$$
I_{0}=\int_{A} \pi(t)^{\xi / q-1} \mathcal{R}_{\sigma} f(t)^{\xi} d t, \quad I_{j}=\int_{A_{\sigma, j}} \pi(t)^{-\frac{r}{n}(1-\varkappa) \xi-1} \varrho_{\sigma}(t)^{\xi} d t .
$$

Using the same methods as in Theorem 1 , we choose $K=\left(n ! 2^{\bar{r}^{\prime}+n+1+1 / \xi}\right)^{q}$ and it only remains to estimate $I_{0}$ and $I_{j}$. Applying Hölder's inequality with exponents $p / \xi$ and $(p / \xi)^{\prime}$, we have (due to $(\xi / q-1)(p / \xi)^{\prime}<-1$ )

$$
I_{0} \leq c\|f\|_{p}^{\xi}
$$


On the other hand, let $\zeta$ be such that

$$
1>\frac{1}{\zeta}= \begin{cases}\frac{\xi}{2} \frac{1-\varkappa}{s} & \text { if } r_{j} \notin \mathbb{N}, \\ \frac{\xi}{2}\left(\frac{1-\varkappa}{s}+\frac{1+\varkappa}{p}\right) & \text { if } r_{j} \in \mathbb{N} .\end{cases}
$$

Then, by Hölder's inequality,

$I_{j} \leq\left(\int_{A_{\sigma, j}} \pi(t)^{-\alpha \zeta^{\prime}} t_{m_{j}}^{\beta \zeta^{\prime}} \frac{d t}{\pi(t)}\right)^{1 / \zeta^{\prime}} \cdot\left(\int_{\mathbb{R}_{+}^{n}} \pi(t)^{-\alpha \zeta} t_{m_{j}}^{-\beta \zeta} \varrho_{\sigma}(t)^{\zeta \xi} \frac{d t}{\pi(t)}\right)^{1 / \zeta} \equiv J_{1} \cdot J_{2}$,

where $\alpha=\frac{r}{2 n}(1-\varkappa) \xi$ and $\beta=\frac{r_{j}}{2}(1+\varkappa) \xi$. The first factor, $J_{1}$, is a constant by the definition of $A_{\sigma, j}$. Indeed,

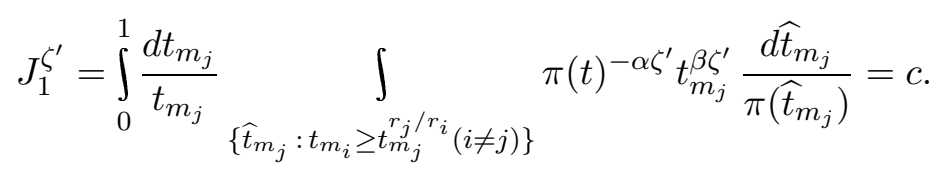

For the second factor, $J_{2}$, we apply Lemma 8 with

$$
\begin{gathered}
m=n, \quad d=1, \quad z_{i}=t_{m_{i}}, \\
a_{i}=\alpha \zeta \quad \text { if } i \neq j, \quad a_{j}=\alpha \zeta+\beta \zeta
\end{gathered}
$$

(as above $\theta_{i}=p$ if $r_{i} \in \mathbb{N}$, and $\theta_{i}=\infty, \phi_{i}=\Omega_{i}$ if $r_{i} \notin \mathbb{N}$ ). Lastly we use the definition of $\Omega_{i}$ and (3.4). So,

$$
J_{2} \leq c\left(\|f\|_{\lambda_{p}^{r_{1}, \ldots, r_{n}}\left(\mathbb{R}^{n}\right)}\right)^{\xi}
$$

and (5.15) is proved.

REMARK 4. As $q$ is not a limit exponent, the embedding

$$
\Lambda_{p}^{r_{1}, \ldots, r_{n}}\left(\mathbb{R}^{n}\right) \hookrightarrow L^{q, \xi}\left(\mathbb{R}^{n}\right)
$$

follows easily from the embeddings without limit exponent for Besov or other spaces [10]. The goal of Theorem 3 is to present a proof based on the $\|\cdot\|_{q, \xi ; \mathcal{R}}$ norm (which is stronger than the usual Lorentz norm if $\xi<q$ ).

\section{References}

[1] C. Bennett and R. Sharpley, Interpolation of Operators, Academic Press, 1988.

[2] O. V. Besov, V. P. Il'in and S. M. Nikol'skiı̌, Integral Representation of Functions and Imbedding Theorems, Vols. 1-2, Winston, Washington DC, and Halsted, New York, 1978.

[3] A. P. Blozinski, Multivariate rearrangements and Banach function spaces with mixed norms, Trans. Amer. Math. Soc. 263 (1981), 149-167.

[4] C. S. Herz, Lipschitz spaces and Bernstein's theorem on absolutely convergent Fourier transforms, J. Math. Mech. 18 (1968), 283-323. 
[5] V. I. Kolyada, On the embedding of the classes $H_{p}^{\omega_{1}, \ldots, \omega_{\nu}}$, Mat. Sb. 127 (1985), 352-383 (in Russian); English transl.: Math. USSR-Sb. 55 (1986), 351-381.

[6] -, On relations between moduli of continuity in different metrics, Trudy Mat. Inst. Steklov. 181 (1988), 117-136; English transl.: Proc. Steklov Inst. Math. 4 (1989), 127-148.

[7] -, Rearrangements of functions and embedding theorems, Uspekhi Mat. Nauk 44 (1989), no. 5, 61-95 (in Russian); English transl.: Russian Math. Surveys 44 (1989), no. $5,73-118$.

[8] - On the differential properties of the rearrangements of functions, in: Progress in Approximation Theory, A. A. Gonchar and E. B. Saff (eds.), Springer, Berlin, 1992, 333-352.

[9] —, On embedding of Sobolev spaces, Mat. Zametki 54 (1993), no. 3, 48-71; English transl.: Math. Notes 54 (1993), 908-922.

[10] - Rearrangements of functions and embedding of anisotropic spaces of the Sobolev type, East J. Approx. 4 (1998), 111-199.

[11] - Embeddings of fractional Sobolev spaces and estimates of Fourier transforms, Mat. Sb. 192 (2001), no. 7, 51-72; English transl.: Sb. Math. 192 (2001), 979-1000.

[12] V. I. Kolyada and F. J. Pérez, Estimates of difference norms for functions in anisotropic Sobolev spaces, Mat. Nachr. 267 (2004), 46-64.

[13] Yu. V. Netrusov, Embedding theorems for the Lizorkin-Triebel classes, Zap. Nauchn. Sem. LOMI 159 (1987), 103-112 (in Russian); English transl.: J. Soviet Math. 47 (1989), 2896-2903.

[14] —, Embedding theorems for spaces with a given majorant of the modulus of continuity, Ph.D. thesis, Leningrad, LOMI AN SSSR, 1988 (in Russian).

[15] S. M. Nikol'skiı̌, Approximation of Functions of Several Variables and Imbedding Theorems, Springer, Berlin, 1975.

[16] P. Oswald, Moduli of continuity of equimeasurable functions and approximation of functions by algebraic polynomials in $L^{p}$, Kandidat thesis, Odessa State Univ., Odessa 1978, (Russian).

[17] A. A. Yatsenko, Iterative rearrangements of functions, and Lorentz spaces, Izv. Vyssh. Uchebn. Zaved. Mat. 1998, no. 5, 73-77; English transl.: Russian Math. (Iz. VUZ) 42 (1998).

Departamento de Matemáticas y Computación

Universidad de La Rioja

Edificio J. L. Vives

Calle Luis de Ulloa $\mathrm{s} / \mathrm{n}$

26004 Logroño, Spain

E-mail: francisco-javier.per@dmc.unirioja.es 